EGU21-4931

https://doi.org/10.5194/egusphere-egu21-4931

EGU General Assembly 2021

(c) Author(s) 2022. This work is distributed under

the Creative Commons Attribution 4.0 License.

\title{
Source apportionment of atmospheric $P$ over East Mediterranean using the Positive Matrix Factorization (PMF) model
}

\author{
Kalliopi Violaki ${ }^{1,2}$, Athanasios Nenes ${ }^{2,4}$, Maria Tsagkaraki ${ }^{3}$, Marco Paglione ${ }^{5}$, Stéphanie Jacquet ${ }^{1}$, \\ Richard Sempere ${ }^{1}$, and Christos Panagiotopoulos ${ }^{1}$ \\ ${ }^{1}$ Aix-Marseille Univ., Université de Toulon, CNRS, IRD, Mediterranean Institute of Oceanography (MIO) UMR 7294, France, \\ CNRS, MIO, France \\ ${ }^{2}$ Laboratory of Atmospheric Processes and their Impacts, School of Architecture, Civil \& Environmental Engineering, École \\ Polytechnique Fédérale de Lausanne, Lausanne, 1015, Switzerland \\ ${ }^{3}$ Chemistry Department, University of Crete, 71003 Heraklion, Crete, Greece \\ ${ }^{4}$ Institute of Chemical Engineering Sciences, Foundation for Research and Technology Hellas, GR-26504, Patras, Greece \\ ${ }^{5}$ Italian National Research Council - Institute of Atmospheric Sciences and Climate (CNR-ISAC), Bologna, Italy
}

The PMF receptor model was applied to a combined dataset using specific markers such as phospholipids and sugars together with other metals (e.g. Al, $\mathrm{Pb}, \mathrm{V}$ ) and ions (e.g. $\mathrm{K}^{+}, \mathrm{Ca}^{2+}, \mathrm{SO}_{4}{ }^{2-}$, $\mathrm{NO}_{3}{ }^{-}$) as tracers of main aerosol sources in order to characterize the sources of $\mathrm{P}$ in atmospheric particles. The samples were collected from East Mediterranean; an oligotrophic region, strongly Plimited, with atmospheric nutrients deposition affecting its primary productivity. The results revealed that dominant sources of $\mathrm{P}$ compounds are the dust (43\%) and the bioaerosols (34\%). The coexistence of these sources in the spring period increased the organic $P$ up to $53 \%$ of total $P$ with more than a half to originate from bioaerosols. Dust is the major source of inorganic $P$ forms with almost equal contribution to the phosphate ions and to the condensed $P$ forms (e.g pyrophosphate or phosphorous minerals).

Based on the results of source apportionment analysis and the atmospheric concentration of $P$ species, the maximum annual deposition scaled to the East Mediterranean surface was $21.5 \mathrm{Gg} P$ with almost equal deposition of org-P and phosphate ions. The soluble $\mathrm{P}$ content from dust aerosols is the similar magnitude of potential bioavailable organic $P$ emitted from bioaerosols $(\sim 4$ $\left.\mathrm{Gg} P \mathrm{y}^{-1}\right)$, especially during the stratification period, when surface water is mostly nutrient starved. Anthropogenic pollution contributes slightly higher to organic P comparing with phosphate ions, while the latter is produced mainly secondary. Biomass burning emissions in the area are associated mainly with the more soluble $\mathrm{P}$. 